\title{
SUBSTITUIÇÃO DO LIGAMENTO DA CABEÇA DO FÊMUR COM AUTO-ENXERTO DE FÁSCIA LATA NA LUXAÇÃO COXOFEMORAL EM CÃES
}

\author{
SUBSTITUTION OF FEMORAL HEAD LIGAMENT IN DOGS WITH FASCIA \\ LATA AUTOGRAFT FOR TREATMENT OF HIP LUXATION
}

\author{
Cláudia Valéria Seullner Brandão ${ }^{1}$ Paulo Iamaguti $^{2}$ \\ Laura Maria Alvarez de Figueiredo ${ }^{3}$
}

RESUMO

Uma técnica cirúrgica para o tratamento da luxação coxofemoral que substituiu o ligamento da cabeça do fêmur por fáscia lata associada ao enxerto ósseo foi realizada em 20 cães. Estes foram separados em cinco grupos e submetidos à eutanásia para realização dos exames macro e microscópicos aos 15, 30, 60, 90 e 120 dias do pós-operatório. Na macroscopia, não foi observada luxação da articulação. Em $65 \%$ dos animais, o enxerto de fáscia lata estava presente. Microscopicamente, a fáscia lata utilizada como substituto do ligamento da cabeça do fêmur não desenvolveu reações inflamatórias, permanecendo preservada e integrada ao tecido ósseo. O uso da fáscia lata como substituto do ligamento da cabeça do fêmur mostrou-se viável, podendo ser utilizada para reforçar a estabilidade articular.

Palavras-chave: cirurgia, articulação, ligamento redondo, histopatologia.

SUMMARY

This study describes a technique for round ligament's substitution by fascia lata and bone autografts. Experiments were carried out in 20 clinical healthy adult mongrel dogs. The animals were divided in five groups, than they were put down for macro and microscopic analysis performed on 15, 30, 60, 90 and 120 days. None of them reluxated the joint. On gross observation, the fascia lata graft was present in $65 \%$ of the dogs. Microscopic examination showed no rejection or inflammatory reactions on fascia lata used as a femoral head ligament substitute. The fascia was preserved and integrated to the bone.

Key words: surgery, joint, round ligament, histopathology.

\section{INTRODUÇÃO}

A luxação coxofemoral é uma afecção traumática freqüentemente encontrada na clínica de pequenos animais, representando 50 a $90 \%$ de todas as luxações articulares (BONE et al., 1984; BRINKER et al., 1986; FOX, 1991; Mc LAUGHLIN, 1995). Várias técnicas cirúrgicas têm sido descritas para restaurar a estabilidade original promovida pelo ligamento da cabeça do fêmur, cápsula articular e tecido periarticular. A reconstrução do ligamento da cabeça do fêmur após a redução cirúrgica da luxação tem sido descrita utilizando-se principalmente materiais sintéticos, como fios de polietileno, náilon, poliéster, fio de aço e pino intramedular de Steinmann ou fio de Kirschner moldado. Essas técnicas citadas necessitam de prévia preparação do material, ou seja, confecção de cavilhas em moldes e a seguir

\footnotetext{
${ }^{1}$ Médico Veterinário, Professor Assistente Doutor, Faculdade de Medicina Veterinária e Zootecnica (FMVZ), Universidade Estadual Paulista (UNESP), Botucatu, Departamento de Cirurgia e Anestesiologia Veterinária, 18618-000, Distrito de Rubião Junior, SP. E-mail: valeria sb@fmvz.unesp.br. Autor para correspondência.

${ }^{2}$ Médico Veterinário, Professor Titular, FMVZ, UNESP, Botucatu, Departamento de Cirurgia e Anestesiologia Veterinária.

${ }^{3}$ Médico Veterinário, Professor Assistente Doutor, FMVZ, UNESP, Botucatu, Departamento de Clínica Veterinária.
} 
esterilização, para posterior utilização no procedimento cirúrgico (KNOWLES et al., 1953; PIERMATTEI, 1963; DENNY \& MINTER, 1973; BRINKER $\boldsymbol{e t}$ al., 1986; BECKHAM et al., 1996; PIERMATTEI \& FLO, 1997; SERDY et al., 1999).

Por outro lado, o transplante da fáscia lata tem sido amplamente descrito na literatura em uma variedade de procedimentos ortopédicos, particularmente reforços e substituições do ligamento cruzado cranial. Mc CURNIN et al. (1971) e BACON et al. (1984) repararam, experimentalmente, o ligamento cruzado utilizando fáscia lata e observaram ligamentos intactos em $61,5 \%$ e $100 \%$ dos animais, respectivamente. BACON et al. (1984), nas análises histopatológicas, confirmaram a manutenção do alinhamento longitudinal do padrão fibrilar e neovascularização destas reconstruções. SILVA et al. (2000), em um experimento semelhante ao de BACON $\boldsymbol{e t} \boldsymbol{a l}$. (1984), observaram, microscopicamente, as fibras da fáscia lata dispostas em feixes paralelos, com aspecto ondulado, envolvido por tecido conjuntivo frouxo. Histopatologicamente, O'DONOGHUE et al. (1966) e BANKS et al. (1985) descreveram um revestimento similar às células da membrana sinovial com neovasos ao redor do ligamento cranial nas substituições realizadas com fáscia lata em cães. SOMER et al. (1990) ressaltaram a invasão de vasos sangüíneos na fáscia lata e tecido de granulação.

O uso de auto-enxertos ósseos apresenta, do ponto de vista anatomofisiológico e funcional, indiscutíveis vantagens com relação aos materiais sintéticos e aloenxertos que podem ocasionar rejeição, infecção e relativa toxicidade. DOBBELAAR (1963) utilizou enxerto ósseo homólogo preservado para realizar acetabuloplastia, estendendo a borda do acetábulo. Para STEVENSON et al. (1996), o auto-enxerto apresenta uma capacidade variável em ativar a formação óssea, induzindo células do tecido conjuntivo a formar tecido ósseo e servindo como substrato para a osteogênese. Além das suas funções biológicas, o enxerto promove suporte estrutural.

O propósito deste trabalho foi avaliar o comportamento anatomopatológico macro e microscópico de uma nova técnica para o tratamento da luxação coxofemoral que substituiu o ligamento da cabeça do fêmur por auto-enxerto de fáscia lata associada ao enxerto ósseo de trocanter maior.

\section{MATERIAL E MÉTODOS}

Foram utilizados 20 cães, sem raça definida, machos e fêmeas, com peso variando de 12 a $32 \mathrm{~kg}$, peso médio de $18 \mathrm{~kg}$, adultos e sadios. Estes foram distribuídos em cinco grupos $(\mathrm{G})$ aleatórios de quatro animais. Os cães dos grupos G15, G30, G60, G90 e G120 foram submetidos à eutanásia, respectivamente, aos 15, 30, 60, 90 e 120 dias após a cirurgia, para realização dos exames anatomopatológicos macro e microscópicos.
Os cães foram tranqüilizados com $1 \mathrm{mg} / \mathrm{kg}$ de clorpromazina ${ }^{a}$ por via intravenosa e anestesiados com pentobarbital sódico ${ }^{\mathrm{b}}$. O acesso dorsal à articulação coxofemoral do membro pélvico esquerdo de todos os cães foi realizado, associandose a osteotomia do trocanter maior do fêmur, segundo técnica descrita por PIERMATTEI (1993), sendo preparada uma tira de fáscia lata $(1$ a $1,5 \mathrm{~cm}$ de largura), a qual foi passada através de um túnel ósseo no fêmur, realizado com furadeira manual e broca de 3,2 milímetros, iniciando na fóvea, tendo continuidade até a face lateral na base do trocanter maior. A seguir, a fáscia lata foi fixada ao fragmento ósseo do trocanter $(18 \times 4 \mathrm{~mm})$ por meio de uma sutura com fio mononáilon 3-0, em pontos do tipo "X" separados. A fáscia e o enxerto corticoesponjoso foram introduzidos até a região retroacetabular através de um orifício realizado no acetábulo, na região de inserção do ligamento da cabeça do fêmur com broca de $4,8 \mathrm{~mm}$. Por tração da fáscia lata, o enxerto foi girado mantendo-se transversal e firmemente ajustado à face medial do acetábulo. A porção emergente da fáscia, próxima ao colo do fêmur, foi suturada à inserção do músculo vasto lateral com ponto do tipo "X" e mononáilon 30, substituindo desta maneira o ligamento (Figura 1). A cápsula articular foi suturada com fio mononáilon 3-0, em pontos do tipo simples separados. A osteotomia do trocanter maior foi reduzida com

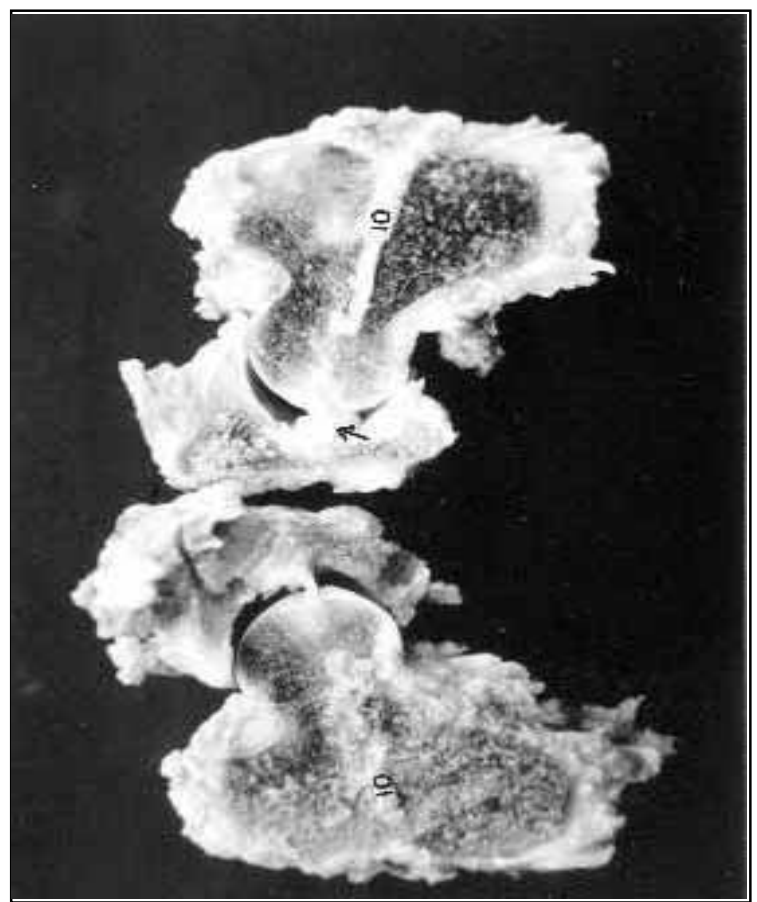

Figura 1 - Aspecto macroscópico da articulação coxofemoral de cão, aos 120 dias do pós-operatório, após a fixação em formol. Notar o enxerto de fáscia lata substituindo o ligamento da cabeça do fêmur (seta) e o trajeto intraósseo (IO) da fáscia lata. 
sutura de fio de aço $\mathrm{n}^{\mathrm{o}} 2$.

Após a cirurgia, o membro operado foi imobilizado com auxílio de uma tipóia do tipo Ehmer com atadura de crepe e esparadrapo, durante dez dias. Os animais receberam duas aplicações de penicilina benzatina $^{c}$ na dose de $20.000 \mathrm{UI} / \mathrm{kg}$ de peso, com intervalo de setenta e duas horas. O antiinflamatório utilizado foi o flunixin meglumine ${ }^{\mathrm{d}}$ na dose de $1 \mathrm{mg} / \mathrm{kg}$ por via subcutânea, durante dois dias. Os pontos da pele foram retirados no décimo dia do pós-operatório. A eutanásia dos animais foi realizada com tiopental sódico.

$\mathrm{Na}$ avaliação macroscópica, a região da articulação coxofemoral esquerda de todos os animais submetidos à eutanásia foi avaliada, observando-se o comportamento do tecido envolvendo o enxerto ósseo, a fixação do enxerto na região retroacetabular, as superfícies articulares, a presença do enxerto de fáscia lata e cápsula articular.

A maceração da articulação coxofemoral esquerda foi realizada em um animal de cada grupo. O membro pélvico esquerdo foi removido juntamente com parte do púbis, ílio, ísquio e acetábulo esquerdos e desarticulado na articulação femorotibial. A pele e a musculatura foram removidas. As peças foram colocadas em cuba de maceração com água corrente durante cerca de oito dias. Em seguida, foram imersas em solução de peróxido de hidrogênio 20 volumes, durante tempo suficiente para o clareamento e expostas ao sol para a secagem e clareamento completo (RODRIGUES, 1973).

$\mathrm{Na}$ avaliação microscópica, todas as articulações coxofemorais operadas, exceto as maceradas, foram avaliadas por meio de microscopia óptica, de acordo com o seu respectivo grupo. Foram examinadas a parte do acetábulo associada ao enxerto ósseo, a cabeça e o colo do fêmur com o trajeto intra-ósseo da fáscia lata e a cápsula articular na região da incisão cirúrgica. A descalcificação foi realizada pelo Método de Cajal, utilizando-se solução aquosa de aldeído fórmico 14\% e ácido nítrico a 3\%. Os fragmentos ósseos, após descalcificados, e a cápsula articular foram submetidos aos processamentos de rotina histológica, corados pelos métodos da hematoxilinaeosina, tricrômico de Masson modificado por Van de Grift e examinados por microscopia óptica.

\section{RESULTADOS E DISCUSSÃO}

O exame macroscópico das articulações mostrou-se satisfatório quanto à manutenção da integridade das superfícies articulares, exceto em um dos cães do G120 que apresentou duas pequenas erosões de três milímetros de diâmetro, na cartilagem da cabeça do fêmur devido a um processo de osteoarite. $\mathrm{O}$ local da incisão capsular apresentou-se espessado devido à proliferação fibrosa cicatricial, não sendo observadas outras alterações na cápsula articular.

Observou-se em $65 \%$ dos animais, presença de uma distinta estrutura fibrosa de coloração branca, ou seja, enxerto de fáscia lata que substituiu o ligamento (Figura 1). Nos relatos de KNOWLES et al. (1953) e LUBBE \& VERSTRAETE (1990) sobre o uso da fáscia lata na articulação coxofemoral, não foi avaliada a presença do enxerto de fáscia, apenas a ausência de luxação. LUBBE \& VERSTRAETE (1990), após a substituição do ligamento da cabeça do fêmur por laçada de fáscia lata, observaram durante a artrotomia exploratória de um caso luxado, que a fáscia lata tinha rompido e desintegrado. Nas substituições do ligamento cruzado cranial em cães por fáscia lata, BACON et al. (1984) observaram $100 \%$ dos enxertos de fáscia lata intactos e Mc CURNIN et al. (1971), em 61,5 \% dos cães, semelhante ao observado neste experimento.

Os animais, sem presença do enxerto de fáscia lata na articulação coxofemoral, mostraram completa reabsorção das porções intra-articulares remanescentes; ao corte longitudinal do fêmur, a fáscia lata permanecia no interior do túnel ósseo realizado. A ausência do enxerto de fáscia lata $(35 \%$ dos animais) não promoveu alteração ou luxação das superfícies articulares. Não foram observadas diferenças na avaliação da locomoção dos animais que apresentaram ou não a presença do ligamento. Alguns fatores, como sutura e reparação da cápsula articular e diminuição da mobilidade inicial pela tipóia do tipo Ehmer, devem ter tido contribuição importante para a ausência de luxação e presença do ligamento em $65 \%$ dos animais.

Uma das razões para a falência de alguns enxertos de fáscia lata pode ser a zona avascular que persiste aproximadamente por oito semanas. $\mathrm{Na}$ literatura consultada, a imobilização pós-operatória, durante um período variável entre duas a quatro semanas e/ou confinamento dos cães em jaulas, foram sempre realizadas nos experimentos com presença de 100\% dos ligamentos (CHIROFF 1975; BACON et al., 1984). O'DONOGUE et al. (1966) relataram que o enxerto autólogo de fáscia lata deve vascularizar-se rapidamente para manter a integridade intra-articular nos casos de ruptura de ligamento cruzado, caso contrário, o mesmo degenera e é reabsorvido. Tal fato pode ter ocorrido nos animais deste experimento que apresentaram ausência do ligamento, associado a excesso de atividade no pós-operatório. Estes, apesar da tipóia, 
eram mantidos soltos no solário de canis coletivos com quatro animais. A ausência do enxerto de fascia lata pode ter sido também, secundária a um eventual erro técnico de posicionamento entre o orifício acetabular e femoral. Forças cortantes repetitivas, com o movimento dorsal do fêmur durante o apoio, podem levar à secção da fascia lata.

A capsulorrafia e reorganização do tecido ao redor da articulação devem ter contribuído para os resultados favoráveis do estudo. BECKHAM et al. (1996) não acreditam que somente a capsulorrafia seja suficiente para manter a redução da luxação nos cães com lesões adicionais e apoio precoce do membro, independente da integridade da cápsula articular reparada.

Nas peças maceradas, as superfícies articulares permaneceram bem definidas, sem alterações das relações articulares. Microscopicamente, aos 15 dias de pós-operatório, observou-se o enxerto de fáscia lata com fibras conjuntivas densas preservadas, envolvido por discreta proliferação de tecido conjuntivo frouxo, neoformação vascular e infiltrado mononuclear. Os ossos do acetábulo e fêmur apresentaram aspectos normais, notando-se atividade de reparação óssea inicial ao redor das superfícies dos orifícios realizados. A fáscia implantada fixava-se ao tecido ósseo por meio de proliferação fibrosa, caracterizada por reação inflamatória entre as fibras conjuntivas, focos de hemorragia, sem interferir com a fáscia e face articular. No enxerto ósseo, um processo inicial de reabsorção óssea foi evidenciado no G30. A membrana sinovial da cápsula articular estava completamente reparada na região da incisão. Aos 30 dias do pós-operatório, a fascia lata apresentava-se preservada, integrada ao tecido ósseo por tecido fibroso com reação inflamatória mais discreta na interface fáscia lata-osso.

Aos 60 dias, uma camada de revestimento multicelular de tecido sinovial envolvendo parcialmente as extremidades intra-articulares do neoligamento de fáscia lata foi observada. Aos 90 e 120 dias, a fáscia lata mostrou-se íntegra e preservada, com organização de fascículos colágenos moderadamente densos, paralelos e compactos com infiltração capilar, sem reação celular e envolvidos por um tecido com maior quantidade de fibras colágenas (Figura 2). Uma camada multicelular de tecido mesenquimal que se assemelhava à membrana sinovial foi observada ao redor do enxerto de fáscia lata. As trabéculas ósseas estavam definidas e a fáscia integrada ao tecido

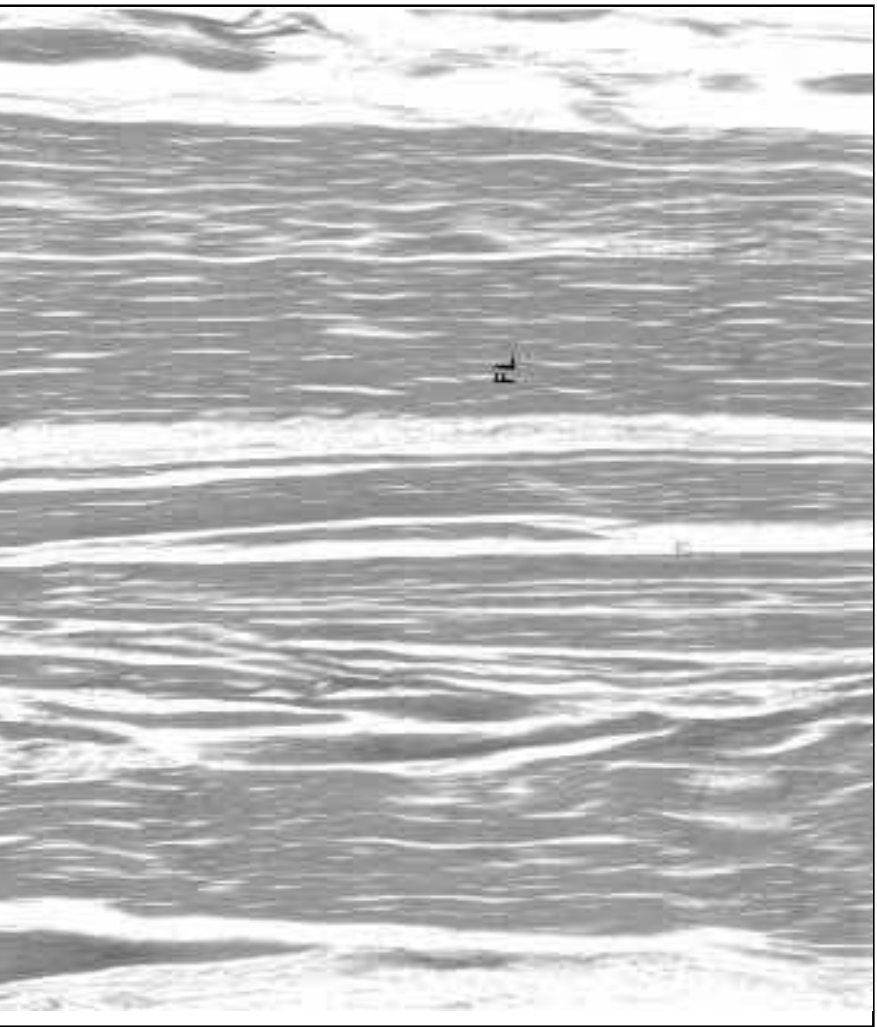

Figura 2 - Secção histológica de uma porção intra-articular do enxerto de fáscia lata (FL) no cão mostrando o alinhamento longitudinal de tecido conjuntivo denso com fibras colágenas orientadas, aos 120 dias de pós-operatório (Tricrômico de Masson modificado por Van de Grift, 100X).

ósseo por um tecido fibroso organizado (Figura 3). Os resultados observados no presente experimento estão de acordo com BACON et al. (1984) e SILVA et al. (2000), que descreveram a presença de fascículos colágenos ao longo da linha natural de tensão do enxerto de fascia lata que substituiu o ligamento cruzado na articulação do joelho. O'DONOGHUE et al. (1966), BANKS et al. (1985) e SOMER et al. (1990) descreveram a presença de revascularização e o revestimento sinovial intraarticular do ligamento cruzado substituído por fáscia lata. A presença de um tecido semelhante ao sinovial foi observado neste experimento, concordando com os resultados descritos.

$\mathrm{Na}$ região do enxerto ósseo foi possível observar pequenos fragmentos ósseos, representando enxerto em amplo processo de reabsorção, substituído quase completamente por tecido conjuntivo denso. DOBBELAAR (1963) observou, também, reabsorção do enxerto cortical homólogo utilizado na acetabuloplastia. O processo lento de reabsorção de tecidos como osso, cartilagem e fáscia, que são compostos por suas respectivas células parenquimatosas envolvidas por grande quantidade de material intercelular, relatado por 


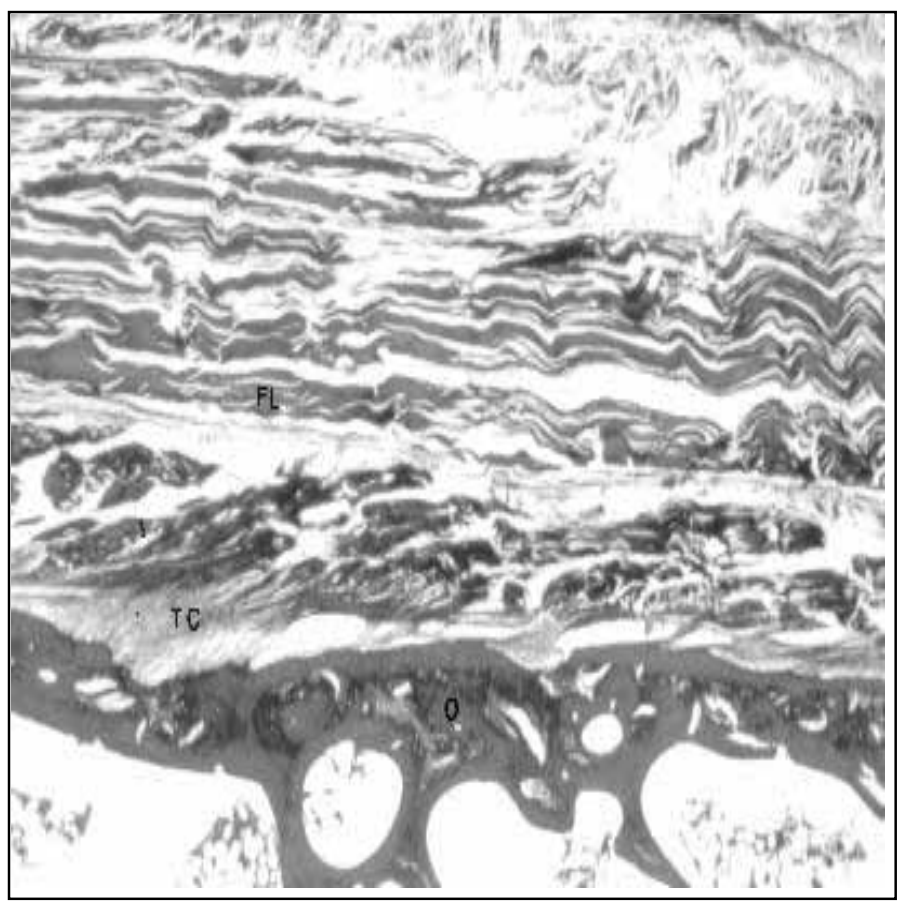

Figura 3 - Secção histológica da união femoral do enxerto de fáscia no cão demonstrando a fáscia lata (FL) íntegra e a superfície óssea reparada $(\mathrm{O})$, com presença de tecido conjuntivo (TC) rico em fibras, interligando as duas estruturas, aos 120 dias do pós-operatório (Tricrômico de Masson modificado por Van de Grift, 32X).

PEER (1951), teve um aspecto positivo nesta técnica, pois esse processo permitiu a união fibrosa da fáscia lata. O enxerto do trocanter maior, apesar de não ter sido incorporado, conseguiu atingir o seu objetivo de fixar a fáscia até que o tecido conjuntivo o envolvesse.

Em um cão, aos 120 dias, observou-se proliferação acentuada de tecido conjuntivo no acetábulo e trabéculas ósseas desorganizadas, áreas de destruição óssea na cabeça do fêmur e hiperplasia vilosa da membrana sinovial. A fáscia manteve-se íntegra e sem sinais de reação inflamatória. Segundo LIPOWITZ (1993), condições que interferem com mecanismos normais da articulação resultam em osteoartrite, observando-se mudanças estruturais na cartilagem, osso e membrana sinovial. Por tratar-se de um caso isolado, com piora súbita dos sinais e ausência de evidências de que a técnica cirúrgica seria a única responsável pelas alterações observadas, acredita-se que houve uma intercorrência externa relacionada a esse animal, como por exemplo, um trauma com deslizamento do membro operado.

A técnica descrita apresenta vantagens com relação à disponibilidade dos materiais empregados, ausência de implante sintético e custos. Quanto às desvantagens, requer uma incisão cirúrgica ampla, imobilização pós-operatória e colocação adequada dos orifícios e enxertos de fascia lata.

Experimentos adicionais, com acompanhamento a longo prazo, ainda são necessários para a utilização da técnica e cães que apresentem luxação coxofemoral traumática.

\section{CONCLUSÃO}

A presença do alinamento fibrilar do ligamento de fácia lata, neovascularização, associado à ausência de reluxação e função locomotora normal na grande maioria dos animais, ajuda a comprovar a eficiência do uso da fáscia lata para substituição do ligamento da cabeça do fêmur e conseqüente reforço da estabilidade nas luxações coxofemorais experimentais e traumáticas.

\section{AGRADECIMENTOS}

Os autores agradecem à FAPESP pela colaboração na execução deste projeto.

\section{FONTES DE AQUISIÇÃO}

a Clorpromazina - Laboratórios Cristália - Rodovia Itapira - Km 14 - Itapira - SP

${ }^{\mathrm{b}}$ Hypnol 3\% - Laboratórios Cristália - Rodovia Itapira - Km 14 Itapira - SP

${ }^{c}$ Benzetacil 600.000 UI - Laboratório Fontoura Wyeth. - Rua Alexandre Dumas, 2200- São Paulo - SP

${ }^{d}$ Banamine injetável - Schering - Plough Veterinária - Estrada dos Bandeirantes, 3091 - Rio de Janeiro - RJ.

\section{REFERÊNCIAS BIBLIOGRÁFICAS}

BACON, M.J.P., PICHLER, M.E., LYND, F.T., et al. Histopathological examination of two cranial cruciate ligaments reconstructions. Journal of American Veterinary Medical Association, Chicago, v.20, n.1, p.65-68, 1984.

BANKS, W.J., PIERMATTEI, D.L., WYKES, P.M., et al. Histological characteristics of fascia lata implants in a synovial environment. Anatomia Histologia Embryologia, Berlin, v.14, n.1, p.79-80, 1985.

BECKHAM, H.P., SMITH, M.M., DOUGLAS, A.K. Use of a modified toggle pin for repair of coxofemoral luxation in the dogs with multiple orthopedic injuries: 14 cases (1986-1994). Journal of American Veterinary Medical Association, Chicago, v.208, n.1, p.81-84, 1996.

BONE, D.L., WALKER, M., CANTWELL, H.D. Traumatic coxofemoral luxation in dogs: results of repair. Veterinary Surgery, Philadelphia, v.13, n.1, p.263-270, 1984. 
BRINKER, W.O., PIERMATTEI, D.L., FLO, G.L. Manual de ortopedia e tratamento das fraturas dos pequenos animais. São Paulo : Manole, 1986. Cap.20: Diagnóstico e tratamento dos estados ortopédicos do membro posterior: p. 282- 293.

CHIROFF, R.T. Experimental replacement of the anterior cruciate ligament. A histological and microradiographic study. Journal of Bone Joint Surgery, Chigaco, v. 57-A, n.2, p.1124-1127, 1975.

DENNY, H.R., MINTER, H.M. Recurrent coxofemoral luxation in the dog. Veterinary Annual, Bristol, v. 14, n.1, p.220225,1973 .

DOBBELAAR, M.J. Dislocation of the hip in dogs. Journal Small Animal Practice, Oxford, v.4, n.2, p.101-109, 1963.

FOX, S.M. Coxofemoral luxations in dogs. Compendium on Continuing Education Practing Veterinary, Trenton, v.13, n.3, p.381-389, 1991.

KNOWLES, A., KNOWLES, J.O., KNOWLES, R.P. An operation to preserve the continuity of hip joint. Journal of American Veterinary Medical Association, Chicago, v.123, n.12, p.508-515, 1953 .

LIPOWITZ, A. J. Degenerative joint disease. In: SLATTER D.H. Textbook of small animal surgery. 2.ed. Philadelphia : Saunders, 1993. Cap.143, p.1921-1927.

LUBBE A.M., VERSTRAETE, F.J.M. Fascia lata loop stabilisation of the coxofemoral joint in the dog and cat. Journal of Small Animal Practice, Oxford, v.31, n.5, p.234$238,1990$.

Mc CURNIN, D.M., PEARSON, P.T., WASS, W.M. Clinical and pathologic evaluation of ruptured cranial cruciate ligament repair in the dog. American Journal Veterinary Research, Schaumburg, v.32, n.10, p.1517-1524, 1971.

Mc LAUGHLIN, R.E. Traumatic joint luxations in small animals. Veterinary Clinics of North American Small Animal Practice, Philadelphia, n.5, v.25, p.1175-1196, 1995.

O'DONOGHUE, D.H., ROCKWOOD, C.A., FRANK, G.R., et al. Anterior cruciate ligament in dogs. Journal of Bone Joint Surgery, Chicago, v.48A, n.3, p.503-519, 1966.

PEER, L.A. The fate of autogenous human bone grafts. British Journal Plastic Surgery, Edinburgh, v.3, n.2, p.233-243, 1951.

PIERMATTEI, D.L. A technique for surgical management of coxofemoral luxation. Veterinary Medicine Small Animal Clinician, Bonner Springs, v.58, n.3, p.373-386, 1963.

PIERMATTEI, D.L. An Atlas of surgical approaches to the bones and joints of the $\mathrm{dog}$ and cats. 3ed. Philadelphia: Saunders, 1993. Cap.6: The pelvis and hip joint: p.222-263.

PIERMATTEI, D.L., FLO, G.L. Brinker, Piermattei and Flo's small animal orthopedics and fracture repair. 3.ed. Philadelphia : Saunders, 1997. Cap.15: The hip joint: p.422468.

RODRIGUES, H. Técnicas anatômicas. Juiz de Fora : UFJF, 1973. Cap.1: Osteotécnicas: p.10-11.

SERDY, M.G., SCHULZ, K.S., HONOF, W.K., et al. Closed toggle pinning for canine traumatic coxofemoral luxation. Veterinary Comparative Orthopaedics Traumatology, Stuttgart, v.12, n.1, p.6-14, 1999.

SILVA, A.M., CARLO, R.J. D., FONSECA, C.C. et al. Aspectos macro e microscópicos da fascia lata utilizada como substituto autógeno do ligamento cruzado cranial. Ciência Rural, Santa Maria, v.30, n.2, p.275-280, 2000.

SOMER, L., VUKADINOVIC, S., SOMER, T., et al. Histologic changes in fascia lata transplants in the replacement of the anterior cruciate ligament of the knee in dogs. Acta Chirurgica Iugoslavic, Skopje, v.37, suppl.1, p.51-55, 1990.

STEVENSON, S., EMERY, S.E., GOLDBERG, V.M. Factor affecting bone graft incorporation. Clinical Orthopedic, Philadelphia, n.324, p.66-74, 1996. 